\title{
Demenzpatienten umfassend behandeln
}

$\mathrm{D}$ emenzerkrankungen können heute zurecht als Volkskrankheit bezeichnet werden. Glücklicherweise nimmt die öffentliche Aufmerksamkeit für diese Krankheiten zu. Leider ist jedoch der Diskurs um wirksame Therapien ebenso wie die Berücksichtigung dieser Krankheitsbilder in der medizinischen Versorgung noch oft geprägt von einer Verdrängung oder Verleugnung der Probleme. Diese erscheinen dann erst recht unlösbar.

Dieses Schwerpunktheft widmet sich nun den Themen, die für eine möglichst lange und gute ambulante Versorgung wichtig sind. Die frühe Beachtung der allgemeinen körperlichen Gesundheit, einer regelmässigen Lebensführung, angemessenen Ernährung und das Erlernen von Strategien zum Umgang mit den Kranken ist entscheidend.

Der Beitrag zu Schlafstörungen stellt dar, dass diese häufig im Krankheitsverlauf auftreten und auf die Zerstörung der neuronalen Grundlagen zirkadianer Rhythmen zurückzuführen sind. Schlafstörungen beeinträchtigen nicht nur die Gesundheit der Demenzkranken, sondern auch ihre Angehörigen und sind nicht ohne Grund Hauptursache für die dann oft notwendige Einweisung in ein Heim.

Bisher wenig beachtet wird auch die Ernährung. Sofern es Untersuchungen gibt, zeigen sie, dass Demenzkranke häufig fehlernährt sind, was den Krankheitsverlauf ungünstig beeinflussen kann und das Entstehen weiterer die Pflege erschwerender Erkrankungen befördern kann. Veränderungen im Geruchssinn, des Geschmacks, des Appetits und der praktischen Nahrungsaufnahme müssen berücksichtigt werden.

Bei aller Diskussion um Sinn und Unsinn von Pharmaka wird oft übersehen, dass es inzwischen gute und wirksame psychosoziale Behandlungsformen gibt, die helfen, Depressionen oder Aggressionen zu vermeiden oder zu mildern. Darüber und vor allem auch die Brückeneffekte - wenn Hilfen für die Kranken auch das Befinden der Angehörigen verbessern - berichtet einer der Beiträge in diesem Heft.

Besondere Bedeutung haben die Angehörigen, die mehrheitlich heute noch die ambulante Versorgung Demenzkranker gewährleisten. Sie möchten einbezogen werden in den Behandlungsprozess. Und uns Ärzten sollte es wichtig sein, ihre Gesundheit mitzubeachten, weil das Risiko pflegebedingter Krankheiten hoch ist. In diesem Heft wollen wir deshalb auch den Angehörigen „das Wort geben“.
Gleichzeitig ist bekannt, dass die familiären Ressourcen in der Zukunft nicht mehr in gleichem Maße zur Verfügung stehen werden wie heute. Alternative Modelle müssen erprobt werden. Die Einbeziehung bürgerschaftlichen Engagements in Form von intra- und intergenerationeller Laienhilfe scheint hier eine Möglichkeit. Die bisherigen Erfahrungen hiermit und was beachtet werden muss, werden in einem weiteren Beitrag dargestellt.

Es ist zu wünschen, dass die Versorgung Demenzkranker nicht nur als Last, sondern auch als befriedigende Aufgabe im (fach-)ärztlichen Alltag selbstverständlich wird. Hierzu sollen die Artikel dieses Schwerpunktheftes ihren Beitrag leisten.

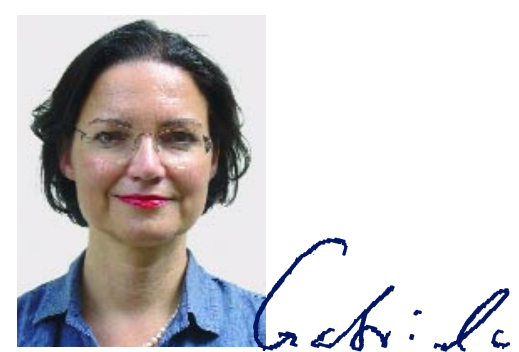

Prof. Dr. med. Gabriela Stoppe, Basel

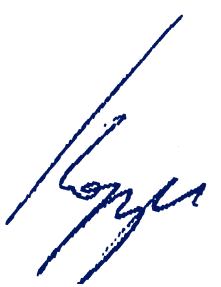

